\title{
Security Systems Based On Eye Movement Tracking Methods
}

\author{
Shaimaa Hameed Shaker \\ Eqbas Ali \\ Israa Ahmed Abdullah
}

\author{
Computer Sciences Department, University of technology \\ Baghdad, Iraq \\ 120011@uotechnology.edu.iq akbas_ali2006@yahoo.com israa_cs93@yahoo.com
}

\begin{abstract}
This survey presents an overview of eye-tracking techniques for various security systems, provides insights into using eye-tracking techniques in security systems. This paper classifies the techniques using some researches. Researches depend on characteristics of eye tracking data, which includes viewer and stimuli aspects, also it does depend on characteristics related to visualization methods. This work contributed in explain some of the security systems that depend on eye gaze tracking, the consequences, the positive and negative aspects resulting from the use of eye gaze tracking in the security system, presents the strengths and weaknesses to keep in mind in future.

The work finally presents a comparison between those security systems for facilitating choose the more accurate and effective system.
\end{abstract}

Keywords. Real-time eye tracking, real-time eye detection, password, gaze, authentication. 


\section{Introduction}

One of the security requirements for general terminal authentication systems is to be easy, fast and secure as people face authentication mechanisms every day and must authenticate themselves using conventional knowledge-based approaches like passwords. But these techniques are not safe because they are viewed by malicious observers who use surveillance techniques such as shoulder-surfing (observation user while typing the password through the keyboard) to capture user authentication data. Also there are security problems due to poor interactions between systems and users. As a result, the researchers proposed eye tracking systems, where users can enter the password by looking at the suitable symbols in the appropriate order and thus the user is invulnerable to shoulder surfing. Eye tracking is a natural interaction method and security systems based on eye movement tracking provide a promising solution to the system security and usability. The aim of this paper is to review techniques or solutions to dealing with eye movement tracking in security systems.

\section{Related Work}

This paper has performed a study of existing security systems that based on eye movement tracking developed by different researchers according to their area of expert. In the following paragraphs are given several of the published researches related to the goals of this work.

- $\quad$ Alexander, Martin and Heinrich (2009) present "Eye-PassShapes Method", EyePassShapes extends and develops two authentication approaches via combining them, PassShapes and EyePIN. In PassShape the users must paint shapes (that consist of strokes) in a certain order, this method increase memorability but doesn't improve security in comparison with PIN or password entry. EyePIN is focused on security instead of usability.
The user's PIN is still the token of authentication, and the security is improved when the input method is changed. Rather than inserting numbers, an eye movement is performed by the the user representing the associated digits. Eye-PassShapes can be considered simpler to be detected than the exact location of the user's look and can work with cheap devices. [1].

- Alain, Sonia and Robert (2010) present "CGP enhancements", CGP which is an abbreviation for Cued Gaze-Points can be considered as a system of graphical pass-word defending from such attacks with the use of eye-gaze pass-word input, rather than mouse-clicks, but it requires certain approaches for improving the accuracy of gaze. This study presented two improvements: a nearest-neighbor gaze-point aggregation method and a one-point calibration prior to entering the pass-word. They reached the conclusion that those improvements made a significant enhancement to the precision of users' gaze and system efficiency [2].

- Justin, Kenrick and Bogdan (2011) present "EyeDent System", which is an improvement to the present authentication systems that depends on eye-tracking which requires pressing a trigger by the user when looking at any symbol. Rather than that, EyeDent, gaze points are being clustered in an automatic way for determining the character chosen by the user; this method is beneficial in allowing the user the authentication at their preferred pace, instead of a predetermined dwell time. In addition, not having visible trigger does not reveal the number of symbols in the pass-word [3]. 
- Andreas, Florian and Albrecht (2012) presented "a novel gaze-based authentication scheme", this scheme uses cued-recall graphical pass-word on all images. This approach uses a computation of visual attention for masking these image parts which will probably be focused on. They created a realistic threat-model concerned with the attacks which could happen in public places, like recording user's actions throughout the process of drawing money from an ATM [4].

- David Rozado (2013) present "the subjectspecific gaze estimation parameters" using this parameter which has been gathered throughout a calibration process to render impractical to another individual to input a password by gazing even in the case where the impostor is aware of the correct password [5].

- Mihajlov, Trpkova and Arsenovski (2013) present "eye tracking study of ImagePass", ImagePass can be considered as a graphical authentication system that is based on recognition. The aim of the study was discovering the users perception and reaction to graphical authentication [6].

- Mohamed, Florian, Mariam, Emanuel, Regina and Andreas (2016) present "Gaze-TouchPass Scheme", it's a multimodal method combining touch and gaze regarding shoulder-surfing resistant authentication in mobiles. GazeTouchPass accepts pass-words with several switches between input modalities throughout the process of authenticating [7].

- Zhenjiang, et al. (2017) present "iType System", a system which utilizes eye gaze to type private input on commodity mobile platforms.
The idea faced 3 main issues: 1) quite low precision of gaze tracking for mobiles; 2) issues in the correction of the input errors because of a lack in comparing of the value the true textentry value; and 3) the movement of the device along with other noises which could be interfering the precision of the gaze tracking and therefore the efficiency of the iType [8].

\section{Typical Model Of Eye Tracking Movement}

Eye tracking can be defined as the procedure of the detection of the eye place throughout video frames for the determination of the position of the gaze. The movement of the eye according to the head could also have some impact [9]. This method will be helpful for disabled people in communicating with their voluntary motions such as movements of eyes and nose. People that have unadorned disabilities could also get benefits from computer access to do their mundane activities such as play games and surf the internet [10]. The concept of eye tracking is used which continuously track the eye movement of a person by using a simple webcam and moves the mouse curser accordingly. The whole process is divided into four stages such as face detection, eyes detection, pupil detection and eye tracking [11] as shown in figure (1). This system utilize a USB (Universal Serial Bus) or builtin camera for capturing and detecting the movements of the user's face [10].

3.1 Face Detection: Face detection is the most important part of the eye tracking process. Featured base and image base methods are the two ways of face detection. [11].

A. Feature-based method: In this method, facial properties are detected (such as, Nose, eyes and so on), then assess their efficiency by observing position and distance from one another. This method can reach high speed in facial detection. Mainly, it is known for its speed pixel precision [10]. 
This technique divided by Hjemal and Low into three categories [15]:

- Low level analysis: It handles segmentation of visual features using pixel properties, grayscale, and animation information.

- Feature analysis: It uses additional knowledge about the face and eliminates ambiguity resulting from low level analysis. The first involves strategies for searching for serial features based on the relative situation of individual facial features. Highlighted facial features are initially identified and allow less visible features.

- Active shape models: Used to make physical and higher level appearance of features. It works in two steps: Look in the image around each point to get a better position for that point, and update the form parameters to better match to these new placements.

B. Image-based Method: This method performs scanning of the particular image with a window looking for faces at every scale and location. By the study of Hjelman this algorithm is essentially just on exhaustive search of the input images for possible face locations at every scale [10]. It contains various approaches such as neural networks, example based learning, support vector machine [15].

3.2 Eye Detection: It consists of four projections for detecting of eye that are Edge-Projection, Luminance Projection, Chrominance Projection, and Final Projection. Viola Jones algorithm is used to use object Detector it is used for detecting object [1]. In the pattern of "Between-theeyes", eyes are found and tracked with updated pattern matching.
After that, from the taken images correct image is chosen based on the distance "Between-the-eyes" [10]. Eye detection approaches [16]:

A. Regression approach: Minimize the distance between the expected and actual eye positions by understanding the job assignment from the image input to the eye sites.

B. Bayesian approach: Learn model of the appearance of the eye and the appearance of non-eye. Use the Baye's principle to build an "eye prospect". Outputs formulas around each pixel of the input image, from which prediction will be extracted.

C. Discriminative approach: The problem is treated as one of the classifications. A classifier is trained to produce a positive result for spots around the eye and negative elsewhere.

3.3 Pupil Detection: In this section the actual pupil of eye is detected first. After detecting eyes it will start its own processing and one type of mark is form on eye portion, after that image is converted into binary form [11].

3.4 Eye tracking: Eye tracking is the last section of this process, in this stage the mouse little bit start moving from its own location. Gradually it starts up its process and start working according to eye movement [11]. Eye tracking techniques [16]:

A. Limbus Tracking: The limbus is the boundary between the white sclera of the eye and the dark iris. Since the sclera is white and the iris is darker, these borders can be easily detected visually as well as tracked. This technique is negatively affected by the eyelid and often hides all or part of the limbus. This makes its uses limited to horizontal tracking. This technique usually does not involve the use of infrared light. 
B. Pupil tracking: There are several reasons for this; but the main advantage is the idea of "bright spot". Such as the red-eye mode when capturing flash images at night, infrared can be used to detect the pupil to create a high intensity bright spot that is easy to find with image processing.
Electrooculography: Depends on electrodes connected to human skin. Because of the high rate of metabolism in the retina compared to cornea, the eye maintains a constant voltage with respect to the retina.

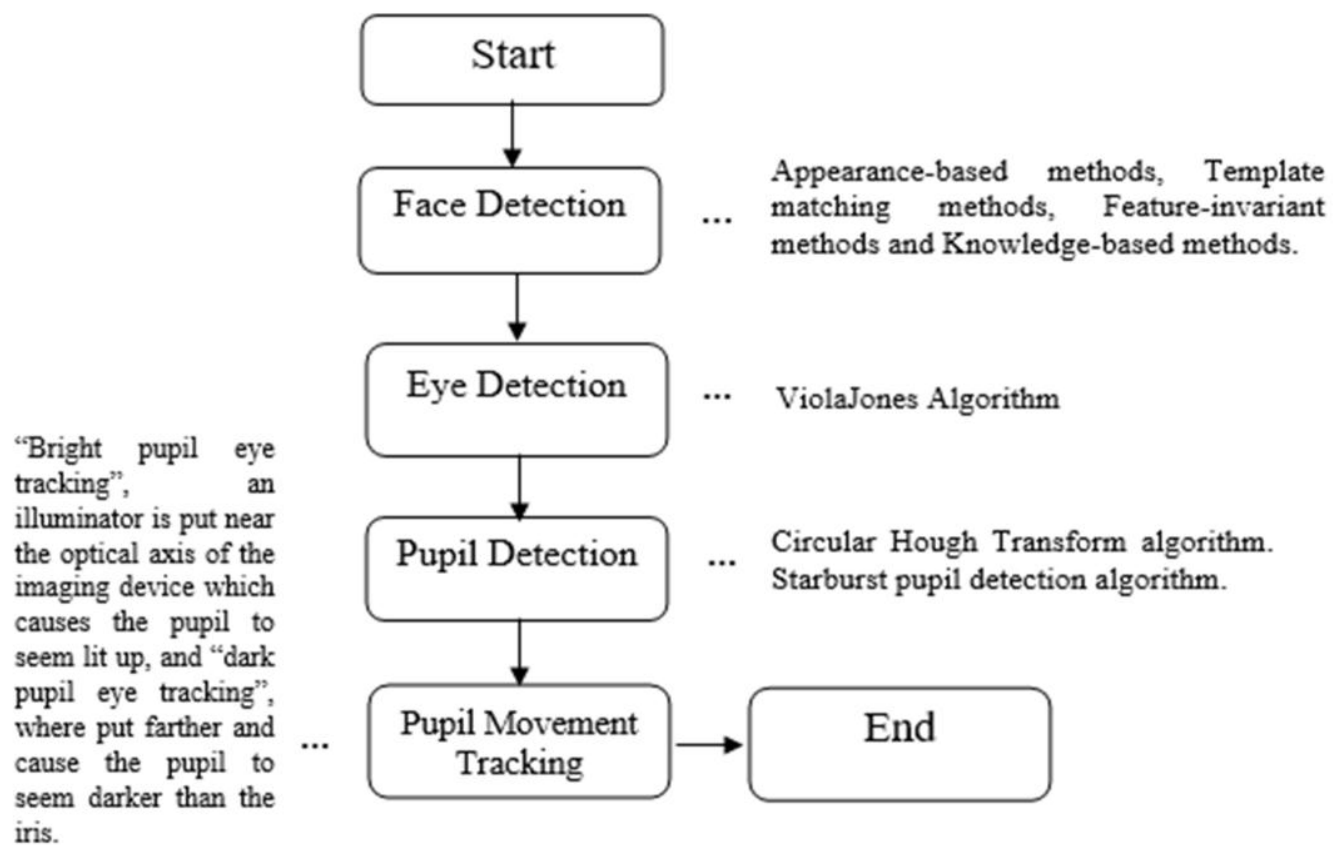

Figure (1): Typical Model eye movement tracking System

4. Basic Measurement Units Used in Pupil Movement Tracking

a) Fixation. It is gathering the gaze points, those points are gathered according to a certain region and time-span. The region of gathering is typically in a range between 20 and 50 pixels, and the time span between 200 and 300 millisecond. Typical fixation measurements are fixation location given as $x^{-}$and $y$ coordinates in pixels, fixation duration in milli-seconds and the fixation count (in other words, number of fixations) [12]. b) Saccade. It describes a fast eye motion from a fixation to another one. Their duration is usually between 30 and 80 milliseconds and are the fastest motion the human can do. The visual information is suppressed during this time span. Usual measurements are the amplitude of the cascade (which is the distance that the saccade has traveled), the duration of the saccade in MS, and the velocity of the cascade in degrees per second [12]. 
Shaimaa .H/Eqbas .A/Israa .A

c) Smooth Pursuit. Throughout the process of presenting dynamic stimuli smooth pursuits could happen. This could be unintentional and only in the case where viewers follow a motion in a presented stimulus. Eye velocity throughout smooth pursuits is in the range between 10 and 30 degrees each second [12].

d) Scan-path. Which is defined as a series of alternating fixations and saccades [12]. Scanpaths explain the path of eye movements on a monitor or a book page [13]. A scan-path can provide data concerning the participant's searching behavior. The optimal scan-path would be a straight line to a certain target. Deviance from this optimal scan-path could be understood as inefficient search [12]. Regarding the efficiency of the task, it has been suggested that the fixations number and duration, and certain scan-paths patterns can be associated with differences in effectiveness in the efficiency of the task [13]. e) Stimulus. It is any visual content which is presented to participants throughout the process of eye tracking. Usually, static and dynamic stimuli, with active or passive content are categorized. Typically, two-dimensional stimuli are shown to participants. Nevertheless, recently, three-dimensional stimuli have also become a focus of study [12].

f) Area of Interest (AOIs), or regions of interest (ROIs) could be defined as parts of a stimulus which are highly important for hypothesis. Generally, depending on the semantic information regarding the stimulus, Area of Interest are being created. A movement from one Area of Interest to another is called a transition. Common metrics of Area of Interest are Area of Interest hit that determines if the fixation is in the Area of Interest or not, the Area of Interest's dwell time in milliseconds, and the transition count (the number of transitions between two Areas of Interest) [12].

g) Blinks. Rapid bilateral eyelid closure and cooccurring eye movement [14].

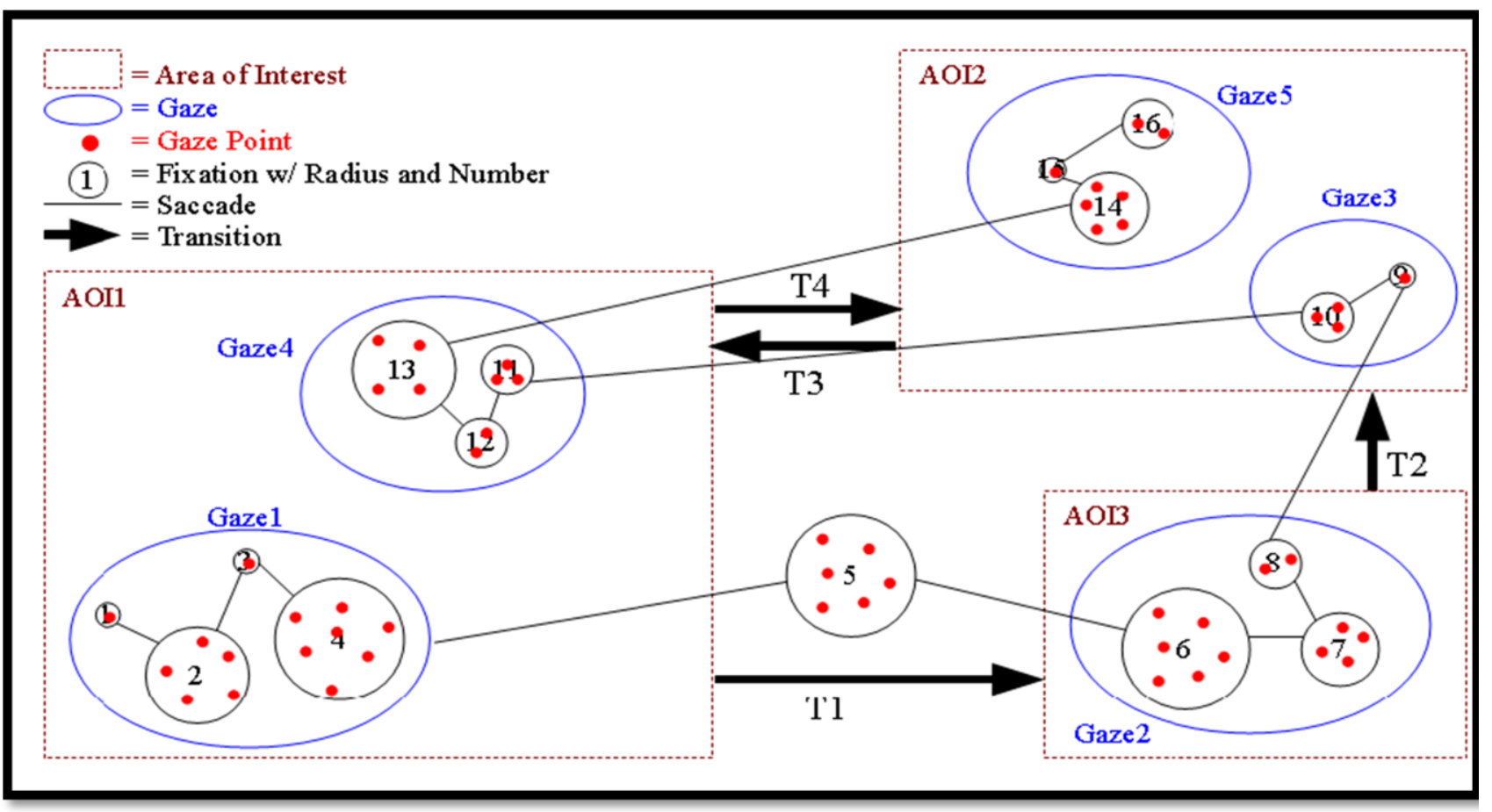

Figure (2): The Gaze points are temporally and spatially aggregated in the fixations. Saccades work as a connection for the fixations, also the fixations have a specific duration that is being represented through radius. A temporal order of fixations is a gaze, but, just if the fixations are within the Area of Interest. An Area of Interest is a region of certain interest on the stimulus. A saccade from one AOI to the next is called a transition. A complete sequence of fixations and saccades is called a scanpath. 


\section{Comparison Among Some Researches:}

This section introduce some details about some works that related of the security system based on eye movement tracking system that shown in table [1].

Table 1: A list concerned with all the references which presented an adoption, enhancement or new existing security systems that based on eye-tracking techniques.

\begin{tabular}{|c|c|c|c|}
\hline Researcher Name & Year & Technique & Details \\
\hline $\begin{array}{l}\text { Alexander, Martin } \\
\text { and Heinrich [1] }\end{array}$ & 2009 & Eye-PassShapes Method & $\begin{array}{l}\text {-EyePassShapes is considered to be simpler to utilize than EyePIN. } \\
\text {-EyePassShapes is considered to be faster than EyePIN. } \\
\text {-EyePassShapes can be considered slower than the standard PIN- } \\
\text { entry. } \\
\text {-EyePassShapes have higher security the than standard PIN-entry. } \\
\text {-EyePassShapes have higher security than the PassShapes. } \\
\text {-PassShapes utilizing EyePassShapes are as memorable as } \\
\text { PassShapes utilizing touchpad. } \\
\text {-PassShapes utilizing EyePassShapes with the repeated input } \\
\text { strategy is considered to be simpler to remember than without. }\end{array}$ \\
\hline $\begin{array}{l}\text { Alain, Sonia and } \\
\text { Robert [2] }\end{array}$ & 2010 & CGP enhancements & $\begin{array}{l}\text {-CGP-2 perform lower rates of error and more success through re- } \\
\text { entering password than CGP-1. } \\
\text {-CGP-2's 1-point calibration retain gaze accuracy at the edge of the } \\
\text { images and not result in more errors at the edges of the images. }\end{array}$ \\
\hline $\begin{array}{l}\text { Justin, Kenrick and } \\
\text { Bogdan [3] }\end{array}$ & 2011 & EyeDent System & $\begin{array}{l}\text {-improvement authentication process by allow single errors such as } \\
\text { a single character missing, inserted, or substituted. }\end{array}$ \\
\hline $\begin{array}{l}\text { Andreas, Florian } \\
\text { and Albrecht [4] }\end{array}$ & 2012 & $\begin{array}{c}\text { a novel gaze-based } \\
\text { authentication scheme }\end{array}$ & $\begin{array}{l}\text {-Image-based graphical passwords are considerably have higher } \\
\text { security than the PIN-based passwords, but it rated as lower } \\
\text { usability. }\end{array}$ \\
\hline David Rozado [5] & 2013 & $\begin{array}{l}\text { the subjectspecific gaze } \\
\text { estimation parameters }\end{array}$ & $\begin{array}{l}\text {-error rates are lower than traditional text based password. } \\
\text {-faster to perform than inputting a password by means of a } \\
\text { keyboard. } \\
\text {-increasing the security by using a database of gaze estimation } \\
\text { models associated to each user. }\end{array}$ \\
\hline $\begin{array}{l}\text { Mihajlov, Trpkova } \\
\text { and Arsenovski [6] }\end{array}$ & 2013 & $\begin{array}{l}\text { eye tracking study of } \\
\text { ImagePass }\end{array}$ & $\begin{array}{l}\text {-through the process of selecting passwords, the Passimage area } \\
\text { have the most recognition with } 55 \% \text { attraction, comes next the } \\
\text { Selected password box with } 25 \% \text { attraction. } \\
\text {-through the process of password confirmation task, Passimage area } \\
\text { have the most recognition with } 65 \% \text { attraction. }\end{array}$ \\
\hline $\begin{array}{l}\text { Mohamed, Florian, } \\
\text { Mariam, Emanuel, } \\
\text { Regina and } \\
\text { Andreas [7] }\end{array}$ & 2016 & GazeTouchPass Scheme & $\begin{array}{l}\text {-especially have security against the side attacks (only } 15 \%-21 \% \\
\text { success rate). } \\
\text {-iterative attacks are considered to be complicated, however they } \\
\text { are possible in ideal conditions }(23 \%-46 \%) \text {. } \\
\text {-authentication time is } 3.1 \text { seconds. } \\
\text {-faster and more secure than gaze-based schemes. }\end{array}$ \\
\hline $\begin{array}{l}\text { Zhenjiang, Mo Li, } \\
\text { Prasant, Jinsong } \\
\text { and Shuaiyu [8] }\end{array}$ & 2017 & iType System & $\begin{array}{l}\text { - address a series of design challenges, covering accuracy, latency } \\
\text { and mobility several aspects. } \\
\text {-high typing accuracy within reasonable short latency in variant } \\
\text { environments. }\end{array}$ \\
\hline
\end{tabular}


According to the comparison, we have graphical passwords and graphical user interface. A quick login and passwords that are easy to use and remember are offered by these systems. These systems face several challenges, one of them gaze accuracy. We found in the paper [2] a solution through 2 recent improvements which increase the accuracy of the gaze without compromising the security and the usability of the system. These enhancements get a good results with low rate of errors. Also we have enhancements in paper [4], but it focused on security and succeed in increase it but rated as low usability. There is also graphical user interface systems, these technique achieved using an eye tracker were shoulder surfing become practically impossible. These system developed in paper [6] and enhancement in paper [3] were it allow single errors such as a single character missing, inserted, or substituted. in these competition there is two systems that depend the method of combining multi-techniques, EyePassShapes in paper [1] get good rates in security and usability but a little slower than PIN-entry, GazeTouchPass in paper [7] that combine between gaze and touch is faster and more secure. in the case of eye tracking there is some enhancements in paper [8] that make eye tracking more efficient in case accuracy and limit some sign in errors, for more secure sign in there is some enhancements in paper [5].

\section{Conclusion}

In this paper, that the techniques was used to obtain the security of different systems was identified depending on the eye pupil tracking, and also the used algorithms were classified to achieve the pupil movement tracking. In addition to that, various measurements were reviewed for the purpose of achieving facial recognition and eye recognition and then distinguish the pupil movement. As well as the show of advantage and disadvantage of the techniques that used to track the eye movement.

\section{References}

[1] De Luca, Alexander, Martin Denzel, and Heinrich Hussmann. "Look into my eyes! Can you guess my password?" Proceedings of the 5th Symposium on Usable Privacy and Security. ACM, 2009.

[2] Forget, Alain, Sonia Chiasson, and Robert Biddle. "Input precision for gazebased graphical passwords." CHI'10 Extended Abstracts on Human Factors in Computing Systems. ACM, 2010.

[3] Weaver, Justin, Kenrick Mock, and Bogdan Hoanca. "Gaze-based password authentication through automatic clustering of gaze points." Systems, Man, and Cybernetics (SMC), 2011 IEEE International Conference on. IEEE, 2011.

[4] Bulling, Andreas, Florian Alt, and Albrecht Schmidt. "Increasing the security of gaze-based cued-recall graphical passwords using saliency masks." Proceedings of the SIGCHI Conference on Human Factors in Computing Systems. ACM, 2012.

[5] Rozado, David. "Using gaze based passwords as an authentication mechanism for password input." 3rd International Workshop on Pervasive Eye Tracking and Mobile Eye-Based Interaction. 2013.

[6] Martin, Mihajlov, Trpkova Marija, and Arsenovski Sime. "Eye tracking recognitionbased graphical authentication." Application of Information and Communication Technologies (AICT), 2013 7th International Conference on. IEEE, 2013.

[7] Khamis, Mohamed, et al. "Gazetouchpass: Multimodal authentication using gaze and touch on mobile devices." Proceedings of the 2016 CHI Conference Extended Abstracts on Human Factors in Computing Systems. ACM, 2016.

[8] Li, Zhenjiang, et al. "iType: Using eye gaze to enhance typing privacy." INFOCOM 2017-IEEE Conference on Computer Communications, IEEE. IEEE, 2017.

[9] Mehrubeoglu, Mehrube, et al. "Real-time eye tracking using a smart camera." Applied Imagery Pattern Recognition Workshop (AIPR), 2011 IEEE. IEEE, 2011. 
[10] Nehete, Minal, Madhuri Lokhande, and Kranti Ahire. "Design an eye tracking mouse." International Journal of Advanced Research in Computer and Communication Engineering 2.2 (2013).

[11] A.V.Bhoyar, SunidhI Sahu and Pooja Padwekar. "EYE TRACKING MOUSE PEOPLE WITH SEVERE DISABILITIES" International Journal of Soft Computing and Artificial Intelligence May-2015.

[12] Blascheck, Tanja, et al. "State-of-the-art of visualization for eye tracking data." Proceedings of EuroVis. Vol. 2014. 2014.

13] Bruneau, Daniel, M. Angela Sasse, and J. D. McCarthy. "The eyes never lie: The use of eye tracking data in HCI research." Proceedings of the CHI. Vol. 2. 2002.
[14] Frank M. Marchak, Ph.D. "Analysis of Eye Movement Data” 2004.

[15] Bakshi, Urvashi, and Rohit Singhal. "A survey on face detection methods and feature extraction techniques of face recognition." International Journal of Emerging Trends \& Technology in Computer Science (IJETTCS) 3.3 (2014): 233-237.

[16] Wankhede, Shrunkhala, S. A. Chhabria, and R. V. Dharaskar. "Controlling mouse cursor using eye movement." International Journal of Application or Innovation in Engineering \& Management 36 (2013): 1-7.

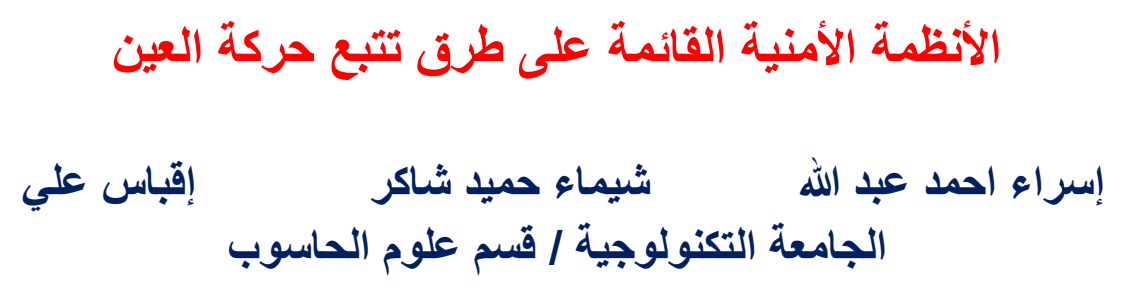

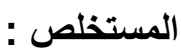

هنالك بعض المزابا و العيوب في جميع طرق تتبع العين، ويعتمد اختيار نظام تتبع العين على الأخذ بعين الاعتبار

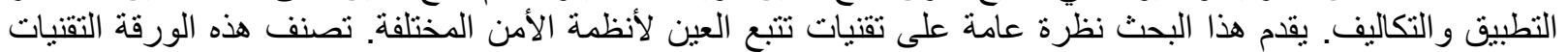

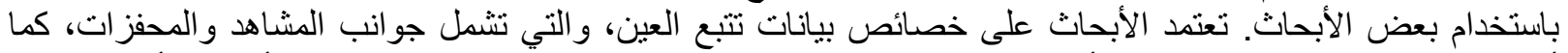

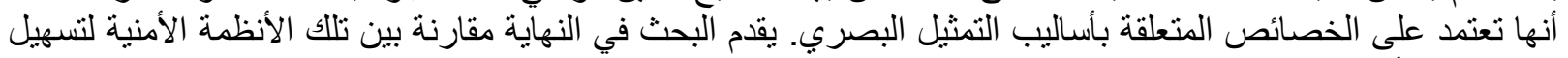
اختيار نظام أكثر دقة وفعالية. 